\title{
What is the mechanism of microalbuminuria in diabetes: a role for the glomerular endothelium?
}

\author{
S. C. Satchell • J. E. Tooke
}

Received: 3 October 2007 / Accepted: 20 December 2007 /Published online: 18 March 2008

(C) The Author(s) 2008

\begin{abstract}
Microalbuminuria is an important risk factor for cardiovascular disease and progressive renal impairment. This holds true in the general population and particularly in those with diabetes, in whom it is common and marks out those likely to develop macrovascular disease and progressive renal impairment. Understanding the pathophysiological mechanisms through which microalbuminuria occurs holds the key to designing therapies to arrest its development and prevent these later manifestations.

Microalbuminuria arises from the increased passage of albumin through the glomerular filtration barrier. This requires ultrastructural changes rather than alterations in glomerular pressure or filtration rate alone. Compromise of selective glomerular permeability can be confirmed in early diabetic nephropathy but does not correlate well with reported glomerular structural changes. The loss of systemic endothelial glycocalyx - a protein-rich surface layer on the endothelium - in diabetes suggests that damage to this layer represents this missing link. The epidemiology of microalbuminuria reveals a close association with systemic endothelial dysfunction and with vascular disease, also implicating glomerular endothelial dysfunction in microalbuminuria.

Our understanding of the metabolic and hormonal sequelae of hyperglycaemia is increasing, and we consider these in the context of damage to the glomerular filtration barrier. Reactive oxygen species, inflammatory cytokines and growth factors are key players in this respect. Taken
\end{abstract}

S. C. Satchell $(\bowtie)$

Academic Renal Unit, University of Bristol, Southmead Hospital, Bristol BS10 5NB, UK

e-mail: s.c.satchell@bristol.ac.uk

J. E. Tooke

Peninsula College of Medicine and Dentistry,

Plymouth, UK together with the above observations and the presence of generalised endothelial dysfunction, these considerations lead to the conclusion that glomerular endothelial dysfunction, and in particular damage to its glycocalyx, represents the most likely initiating step in diabetic microalbuminuria.

Keywords Diabetes · Glomerular endothelial cell . Glomerular filtration barrier . Glycocalyx . Microalbuminuria $\cdot$ Podocyte

$\begin{array}{ll}\text { Abbreviations } \\ \text { CRP } & \text { C-reactive protein } \\ \text { GBM } & \text { glomerular basement membrane } \\ \text { GFB } & \text { glomerular filtration barrier } \\ \text { HSPG } & \text { heparan sulphate proteoglycan } \\ \text { eNOS } & \text { endothelial nitric oxide synthase } \\ \text { NFkB } & \text { nuclear factor } \mathrm{kB} \\ \text { ROS } & \text { reactive oxygen species } \\ \text { VEGF } & \text { vascular endothelial growth factor } \\ \text { vWF } & \text { von Willebrand factor }\end{array}$

\section{Introduction}

The associations between microalbuminuria, cardiovascular disease and progressive renal impairment are well described, but how these are linked mechanistically is something of a conundrum [1]. Here we focus on the pathogenesis of microalbuminuria in patients with diabetes, in whom it occurs commonly and has particular significance. In type 1 diabetes the prevalence gradually increases from onset of disease ( $6 \%$ after $1-3$ years), reaching over $50 \%$ after 20 years [2]. In type 2 diabetes the prevalence is $20-25 \%$ in both newly diagnosed and established diabetes [3]. However, 
it is also instructive to review the general epidemiology of microalbuminuria, including those conditions with which it is associated and those for which it is a risk factor. Such an analysis reveals generalised endothelial dysfunction as a common denominator in microalbuminuria in both the general and diabetic populations. In 1989, this observation led to the hypothesis that a common process underlies both microalbuminuria and generalised endothelial dysfunction in diabetes. This process was suggested to be the dysregulation of enzymes involved in metabolism of extracellular matrix, the 'Steno hypothesis' [4].

Nearly 20 years on from the Steno hypothesis, the determinants of selective glomerular permeability to proteins at the cellular and molecular level are much better understood. In particular, the importance of podocytespecific proteins in the regulation of selective permeability has been recognised. Similarly, much is now known about the biochemical derangements important in the pathogenesis of diabetic complications. We draw together these elements to consider the pathophysiological mechanisms through which diabetes exerts its effects on glomerular permeability in the initiating stages of diabetic nephropathy, i.e. at or before the appearance of microalbuminuria. These early changes establish the milieu in which the more advanced changes of overt diabetic nephropathy develop. Defining the mechanistic links from biochemical derangements to the appearance of increased urinary albumin highlights key elements in the pathophysiological pathway of the development of both diabetic nephropathy and micro- and macrovascular disease elsewhere.

We hold with the established view that increased transglomerular passage of albumin is the major source of microalbuminuria [5]. While other hypotheses have been advanced, for example, failure of tubular reuptake of albumin, none are sufficiently robust to seriously challenge this position.

\section{In both general and diabetic populations, conditions associated with endothelial damage predispose to microalbuminuria}

In the general (non-diabetic) population, hypertension is the major risk factor for microalbuminuria, and the prevalence of microalbuminuria in essential hypertension is around $25 \%$. Individuals with essential hypertension who develop microalbuminuria have a higher incidence of biochemical disturbances, implying that hypertension per se may not be the cause of microalbuminuria, but, rather, these additional derangements [6]. Microalbuminuria is strongly associated with vascular disease in hypertensive patients, suggesting that it is a marker of vascular and/or endothelial damage in this condition [7].
The insulin resistance syndrome describes a clustering of disorders the underlying pathology of which is thought to be related to insulin resistance and/or endothelial dysfunction [8]. Microalbuminuria is associated with several of the disturbances found in the insulin resistance syndrome, including endothelial dysfunction and obesity, in addition to type 2 diabetes. Proinflammatory cytokines produced by visceral adipocytes (adipokines) have recently emerged as important mediators of the increased cardiovascular risk associated with the insulin resistance syndrome. These adipokines represent a possible link from insulin resistance and obesity to microalbuminuria in the non-diabetic population.

Microalbuminuria can be detected in patients undergoing major surgery, particularly when complicated by sepsis [9], and is associated with other inflammatory states, including rheumatoid arthritis and inflammatory bowel disease [10]. Microalbuminuria can also be detected in a significant proportion of the normal non-diabetic, normotensive population (6.6\% in one study [11]), where it also associates with cardiovascular disease. Male sex [11] and hormone replacement therapy in women [12] seem to increase susceptibility to microalbuminuria, and although the basis for this is not clear, the fact that men have a higher incidence of vascular disease in general implies a common aetiology.

Hypertension is approximately twice as frequent in individuals with diabetes as in those without, and hypertensive individuals are predisposed to the development of diabetes [13]. Hypertension is certainly a major determinant of microangiopathy in diabetes, but the relationship between hypertension and microalbuminuria in diabetes is complex. Hypertension and microalbuminuria often coexist in diabetic patients, and reducing blood pressure reduces microalbuminuria in type 1 diabetes [14]. However, it is unclear whether hypertension contributes to the development of microalbuminuria in diabetes. At least in type 1 diabetes, hypertension and microalbuminuria appear to develop together: in longitudinal studies there is no evidence that hypertension develops before microalbuminuria [15]. This is more difficult to demonstrate in type 2 diabetes, perhaps because of the heterogeneity of the disease.

One third of patients with type 1 diabetes develop advanced nephropathy, and the renal status of probands of diabetic patients makes a difference of nearly $50 \%$ in risk [16]. A family history of hypertension also predisposes to microalbuminuria in diabetes [17]. These observations suggest a genetic predisposition in individuals with type 1 diabetes who develop diabetic nephropathy, but as yet no gene has been identified. Perhaps most significant is that this and other evidence suggests that the susceptibility of type 1 diabetic patients to diabetic nephropathy is increased in response to increased genetic risk of insulin resistance [18]. Consequently, it is the combination of this genetic risk 
with hyperglycaemia and its sequelae that leads to microalbuminuria and, eventually, diabetic nephropathy.

Thus, in the diabetic as well as in the general population the risk factors for the development of microalbuminuria can be grouped into those associated with vascular disease, including endothelial dysfunction, inflammation and insulin resistance. This implies that microalbuminuria may also, at least in these situations, result from endothelial dysfunction. This does not preclude significant damage to other components of the vessel wall, including basement membrane, pericytes, matrix components (collagen, elastin) and vascular smooth muscle cells. However, as the principal regulators of vascular permeability, endothelial cells are likely to be involved in systemic increases in permeability.

Conditions associated with endothelial damage predispose individuals to microalbuminuria.

\section{Microalbuminuria is a risk factor for macro- and microangiopathy, including advanced diabetic nephropathy}

Microalbuminuria is a significant risk factor for cardiovascular mortality in type 1 [19] and 2 diabetes [20], as well as in the non-diabetic population [21]. In type 1 diabetes, microalbuminuria is closely associated with microangiopathy elsewhere (e.g. retinopathy). Although this association is also present in type 2 diabetes, in a proportion of these patients microalbuminuria does not appear to reflect generalised microvascular damage [22].

Microalbuminuria predicts the development of overt diabetic nephropathy in type 1 and 2 diabetes; however, the relationship in type 2 diabetes is less clear because of the greater heterogeneity of this condition and the presence of other risk factors for microalbuminuria in these, usually elderly, patients [23]. Microalbuminuria invariably precedes overt diabetic nephropathy, and although microalbuminuria may regress spontaneously in a proportion of cases, it remains the best documented predictor for high risk of development of diabetic nephropathy in both type 1 and type 2 diabetes [24].

Microalbuminuria is a risk factor for vascular disease and nephropathy.

The relationship between microalbuminuria and vascular disease suggests a common causality, as established risk factors explain at most a small part of these associations [25]. Unifying mechanisms, such as generalised endothelial dysfunction or inflammation, are therefore implicated [8].
Indeed, AER is correlated with endothelial dysfunction in type 1 and type 2 diabetes, and in the non-diabetic population [26]. Markers of chronic low-grade inflammation, including $\mathrm{C}$-reactive protein (CRP), are also correlated with microalbuminuria in type 1 and type 2 diabetes [27], and increasing evidence from the non-diabetic population indicates the importance of inflammation in the pathogenesis of cardiovascular disease [28].

\section{Generalised and glomerular endothelial dysfunction}

Markers of endothelial dysfunction, including elevated serum von Willebrand factor (vWF) and increased transcapillary albumin escape rate, are present before the onset of microalbuminuria in type 1 diabetes and worsen in association with it [27, 29]. Type 2 diabetes is often complicated by the presence of other risk factors for vascular disease, and discerning the contribution of hyperglycaemia and its sequelae to endothelial dysfunction in this condition is more difficult. While in some type 2 diabetic patients microalbuminuria may occur in the absence of evidence of endothelial dysfunction, in others, vWF levels predict its development [30]. The close association between endothelial dysfunction and microalbuminuria in type 1 diabetes may underlie the predictability of development of diabetic nephropathy and the greater susceptibility to micro- and macrovascular disease in other organs.

Endothelial dysfunction precedes microalbuminuria.

As endothelial dysfunction is an important antecedent of microalbuminuria in both types of diabetes (albeit with a less predictable relationship in type 2 ), it provides an attractive explanation for the association between microalbuminuria and vascular disease in diabetes (Fig. 1). But to what extent can endothelial dysfunction be said to cause microalbuminuria? As the glomerular endothelium is exposed to the same diabetic milieu as other endothelia, it is highly likely that it is also dysfunctional. This begs the question of how glomerular endothelial dysfunction could lead to microalbuminuria. To address this we now turn to consider the structure and function of the glomerular filtration barrier (GFB).

\section{The glomerular filtration barrier is a complex biological sieve}

Unlike other capillaries, glomerular capillaries have a high permeability to water (hydraulic conductivity) yet, like 


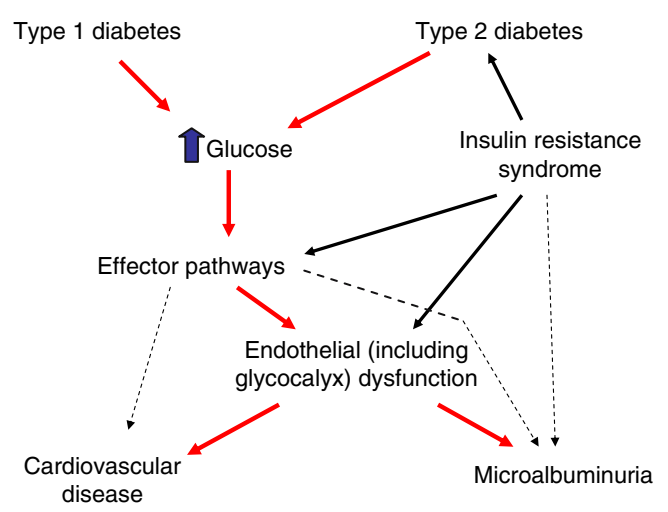

Fig. 1 The relationship between hyperglycaemia, insulin resistance, endothelial dysfunction, macrovascular disease and microalbuminuria in type 1 and type 2 diabetes. Proposed major pathways are represented by red arrows; those of less certain significance by black arrows. The diagram illustrates, for the example, a possible mechanism for the increased risk of microalbuminuria in patients with type 1 diabetes and a susceptibility to insulin resistance. Particularly in type 2 diabetes, other pathways, not directly involving endothelial dysfunction, are likely in the pathogenesis of macrovascular disease and may also contribute to microalbuminuria (broken arrows)

other capillaries, are relatively impermeable to macromolecules. These fundamental permeability properties depend on the unique, three-layer structure of the GFB: the endothelium with its glycocalyx, the glomerular basement membrane (GBM) and podocytes (glomerular epithelial cells; Fig. 2) [31].

Glomerular endothelial cells Glomerular endothelial cells are highly specialised cells with regions of attenuated cytoplasm punctuated by numerous fenestrae, circular transcellular pores $60-80 \mathrm{~nm}$ in diameter [31,32]. Initially these fenestrations were thought of as empty and to therefore provide little barrier to the passage of proteins. Standard fixation protocols for electron microscopy do not preserve the glycocalyx, but newer fixation techniques have allowed the demonstration of a glomerular endothelial glycocalyx of $200-400 \mathrm{~nm}$ in thickness [33, 34]. This glycocalyx covers both fenestral and inter-fenestral domains of the glomerular endothelial cell luminal surface.

Studies of the systemic endothelial glycocalyx are instructive here. The glycocalyx is a dynamic, hydrated layer largely composed of glycoproteins and proteoglycans with adsorbed plasma proteins. Heparan sulphate proteoglycans (HSPGs) are largely responsible for the negative charge characteristics of the glycocalyx. Removal of the glycocalyx increases vascular protein permeability, providing evidence that it hinders the passage of macromolecules $[35,36]$. Compared with those without, capillaries with fenestrations are much more permeable to water and small solutes, but not to proteins. These characteristics can only be explained by the glycocalyx [37].
Therefore, the presence of a significant glomerular endothelial glycocalyx implies that the glomerular endothelium significantly contributes to the barrier to macromolecules [31, 32, 38]. Experimental data support this view: in mice treated with glycocalyx-degrading enzymes the distance between luminal lipid droplets and the glomerular endothelium was decreased, and this was accompanied by an increase in AER [39]. In rats, under normal perfusion conditions albumin is confined to the glomerular capillary lumen and endothelial fenestrae [40]. Endothelial glycocalyx has the correct anatomical distribution (on the surface of endothelial cells, including in fenestral openings) to explain this distribution. Reactive oxygen species (ROS), which are known to disrupt the glycocalyx [41], cause torrential proteinuria without any identifiable structural changes in the GFB using standard electron microscopy techniques [42]. Furthermore, the ability of human glomerular endothelial cell glycocalyx to form a permeability barrier to macromolecules can be directly demonstrated in vitro [43].

Endothelial glycocalyx forms a barrier to protein permeability in both systemic and glomerular capillaries.

The GBM The GBM is a basal lamina specialised for the structural requirements of the GBM and its filtration function. It is a hydrated meshwork of collagens and laminins to which negatively charged HSPGs are attached. Traditional concepts have therefore characterised the GBM as a charge-selective barrier. However, more recent analyses indicate that it only makes a small direct contribution to the barrier to protein passage [31].

Podocytes Podocytes, or, more specifically, their interdigitating foot processes, form the outer layer of the GFB (Fig. 2). The gaps between adjacent foot processes, the 'filtration slits' $(25-60 \mathrm{~nm})$, are spanned by the slit diaphragm. This is a molecular structure thought to form the most restrictive barrier to the passage of water and macromolecules. The effect of mutations in podocyte-specific proteins (e.g. nephrin mutations result in congenital nephrotic syndrome) indicate the importance of podocytes in resisting the passage of protein [44]. However, exactly how podocytes and their foot processes contribute to selective permeability is not yet clear. Electron microscopy studies have suggested that the slit diaphragm has a porous structure with a pore half-width of $2 \mathrm{~nm}$. However, this figure is too small to account for the experimentally observed passage of solutes of various radii, emphasising that our understanding of slit diaphragm function is incomplete [45]. Podocyte biology has been reviewed in detail elsewhere [44]. 
Fig. 2 Representation of a cross-section through the GFB showing the three-layer structure consisting of glomerular endothelium and glycocalyx, glomerular basement membrane (GBM) and podocyte foot processes. Albumin, represented by orange ellipses, does not pass through the normal GFB in significant amounts

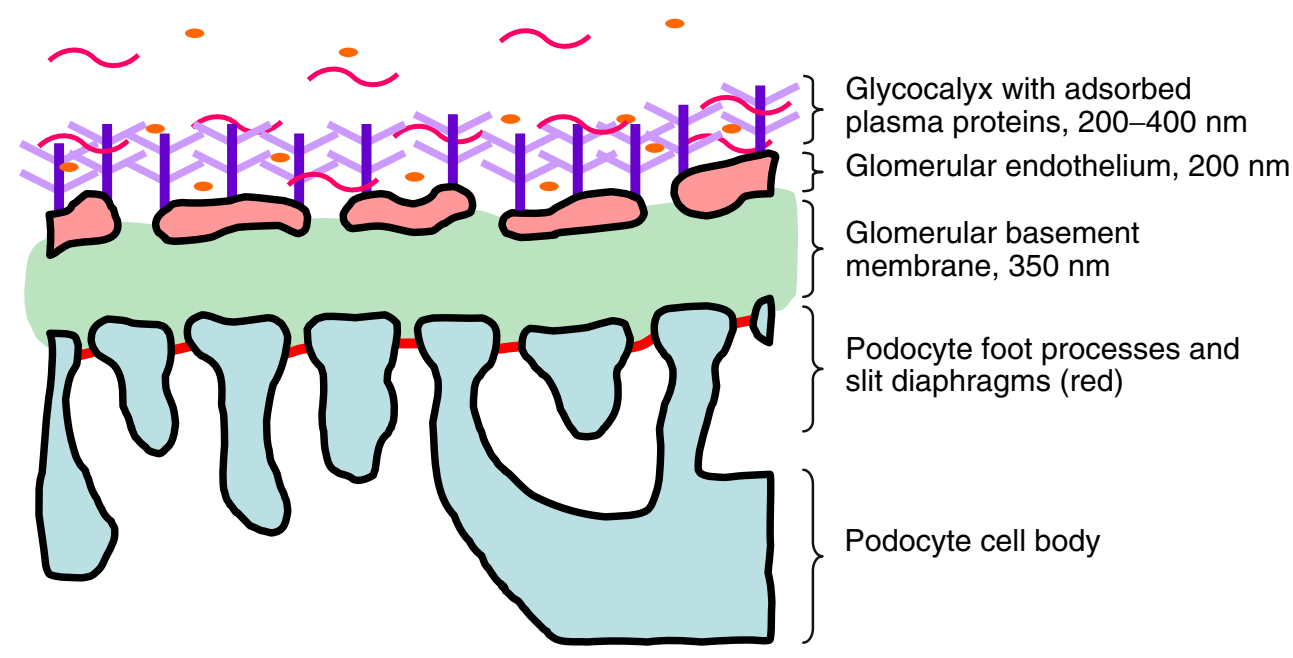

\section{Passage of albumin across the normal GFB}

Filtration Water filters across the GFB via gaps through or between cells rather than through the cell cytoplasm. Therefore, the high hydraulic conductivity of the GFB depends on the presence of fenestrae and filtration slits. Fifty per cent of the hydraulic resistance of the GFB is afforded by the two cell layers (endothelium and podocyte foot processes), and 50\% by the GBM [46].

Solute flux Solute flux (including albumin) from the glomerular capillary occurs by a combination of convection (i.e. being swept along by the filtration of water) and diffusion [31]. The rate of convection of a solute depends on the filtration rate and on its reflection coefficient. Reflection coefficients are related to solute size, larger solutes having higher reflection coefficients. A value of 1 indicates that a molecule is totally excluded. The rate of diffusion of a solute depends on the concentration gradient and its diffusivity (solute permeability). The diffusivity of a solute decreases with increasing size. A measure of overall flux of a solute is given by the sieving coefficient: the filtrate to plasma concentration ratio. The sieving coefficient for albumin across the normal GFB is $<0.001$ [47]. The ratio of the contribution of convective to diffusive flux (the Peclet number) increases with increasing molecular size. That is, for small molecules, diffusion dominates; for larger molecules, convection dominates. The Peclet number for albumin across the normal GFB is near to unity, so both diffusion and convection contribute [48]. Hence, an increase in GFR will result in an increase in the convective flux of albumin. However, despite this, the best estimates indicate that even a $50 \%$ increase in filtration rate would only increase urinary albumin in the sub-microalbuminuric range, as the majority of the excess albumin is reabsorbed by the tubules [47]. Therefore, for albumin flux to increase sufficiently to produce microalbuminuria (assuming normal tubular reuptake), the
GFB must be physically altered in such a way as to increase the sieving coefficient of albumin across it.

Models have been developed to correlate the macromolecular sieving properties of the GFB with the structure and properties of its components [31]. These indicate that the GFB functions as a whole, with each layer having an important contribution to selective permeability. Whereas hydraulic resistances are essentially additive, sieving coefficients are multiplicative. This relationship means that a change in one element significantly affects the overall protein permeability to the same degree, i.e. a $10 \%$ change in permeability of any one layer will produce a $10 \%$ change in overall GFB permeability. Importantly, this means that, regardless of which layer is most restrictive, a change in the permeability of any layer of the GFB could potentially account for microalbuminuria.

The presence of microalbuminuria implies dysfunction of the glomerular filtration barrier.

Theoretically, this could result from damage to any of its layers, including the endothelial glycocalyx.

\section{Structural alterations in the GFB associated with microalbuminuria in diabetes}

Glomerular structural changes typical of diabetic nephropathy are commonly established by the time microalbuminuria becomes apparent [49, 50]. However, the changes seen are heterogeneous, and all may be found in normoalbuminuric diabetic patients [51]. Early changes described include an increase in glomerular size, GBM thickening, mesangial expansion and broadening of podocyte foot processes [49-51]. The increase in glomerular size is due both to mesangial expansion and to enlargement in glomerular capillaries. The latter occurs at least in part 
through angiogenesis, an endothelium-dependent process $[52,53]$. Glomerular structural changes are less marked in type 2 diabetes, with only a third conforming to the classical pattern observed in type 1 diabetes [22].

Specific assessment of structural changes in glomerular endothelial cells and associated glycocalyx in diabetes has not been performed. Evidence is emerging, however, that total systemic glycocalyx volume is reduced by acute hyperglycaemia in humans [54]. Furthermore, type 1 diabetic patients have decreased systemic glycocalyx volume, and this correlates with the presence of microalbuminuria [55]. GBM thickening alone, without change in composition, does not significantly affect its protein permeability characteristics.

Generalised glycocalyx damage occurs in diabetes and is associated with microalbuminuria.

Broadening of podocyte foot processes (effacement) is an indicator of podocyte injury but generally correlates poorly with degree of proteinuria [56]. Indeed, proteinuria may occur in the complete absence of structural changes to podocytes [57]. This is the case with diabetic microalbuminuria, which can occur in the absence of such changes, at least in type 2 diabetes [58]. Some have suggested that podocyte loss occurs in early diabetes and that this would contribute to the filtration barrier defect [51]. Others have argued that significant early podocyte loss does not occur [59]. A likely reconciliation is that the lower proportion of podocytes seen in early disease is due to a relative increase in mesangial and endothelial cells, while podocyte loss occurs at a later stage.

Recognised structural changes in the glomerular filtration barrier in diabetes do not correlate well with microalbuminuria

\section{Functional alterations in GFB selective permeability associated with microalbuminuria in diabetes}

Increased flux of albumin across the GFB in diabetic microalbuminuria can be confirmed in experimental models using isolated glomeruli [60] and by inhibition of tubular reabsorption of albumin [61]. Analysis of the permeability of the GFB to molecules of varying size and charge can be used to estimate whether the increased flux of albumin is due to loss of size or charge selectivity of the GFB. In animal models of diabetes the defect is primarily in charge selectivity [61]. In clinically healthy non-diabetic individuals with microalbuminuria, loss of both size and charge selectivity of the GFB can be demonstrated [62]. However, in type 1 diabetes, defects in charge selectivity occur earlier than loss of size selectivity [63]. Similarly, in Pima Indians with type 2 diabetes, microalbuminuria is associated with loss of charge selectivity. Loss of size selectivity is seen only in those developing macroalbuminuria [58].

Defects in charge selectivity predominate over defects in size selectivity in diabetic microalbuminuria, consistent with glycocalyx damage.

We have already noted that physical alteration of the GFB is necessary to increase its permeability to albumin. Therefore, this confirmation of changes in GFB selective permeability in diabetic microalbuminuria in the absence of clearly identified structural correlates suggests that the key changes are yet to be elucidated. These considerations and the predominance of defects in charge selectivity point to alterations in the negatively charged glomerular endothelial glycocalyx as the missing link. We now move to consider what aspects of the diabetic milieu are responsible for these GFB changes and how they might cause endothelial, including glycocalyx, dysfunction.

\section{Metabolic pathways and effectors from hyperglycaemia to microalbuminuria}

There is now overwhelming evidence that hyperglycaemia is the major initiating factor in the pathogenesis of diabetic complications, including microalbuminuria. However, most adverse effects of glucose are mediated indirectly through diverse metabolic pathways. Four major hypotheses have highlighted the roles of AGEs, increased activity of the polyol pathway, activation of protein kinase $\mathrm{C}$ and increased flux through the hexosamine pathway (Fig. 3). Activation of these pathways in turn causes dysregulation of a number of effector molecules which cause cellular damage and dysfunction. The roles of these pathways and effectors have been studied in detail in overt diabetic nephropathy, but the importance of these elements individually in its initiation and the appearance of microalbuminuria are less clear.

For example, TGF $\beta$ is an effector molecule with a clear role in the progression of established diabetic nephropathy, but there is no evidence that it is important in the mechanism of proteinuria per se. It is a key pro-fibrotic mediator, and in type 1 and type 2 diabetes, TGF $\beta$ is elevated in serum, urine and glomeruli from an early stage of disease. Its levels correlate with the degree of mesangial expansion, interstitial fibrosis and renal insufficiency, but not with microalbuminuria. Similarly, in animal models, blockade of TGF $\beta$ signalling inhibits the development of these pathological features, apart from the microalbuminuria [64, 65].

Here we focus on selected intermediaries and effectors that have an identifiable role in microalbuminuria. In large 
Fig. 3 Pathways to microalbuminuria in diabetes. Hyperglycaemia, through increased mitochondrial superoxide production, dysregulates key intracellular metabolic pathways. These in turn lead to the production of effectors that directly cause glomerular endothelial cell (GEnC) dysfunction (particularly of the glycocalyx) and disturb podocyte-endothelial cell communication. This results in microalbuminuria. Progression of these lesions and development of other glomerular changes, including podocyte damage, lead to overt diabetic nephropathy

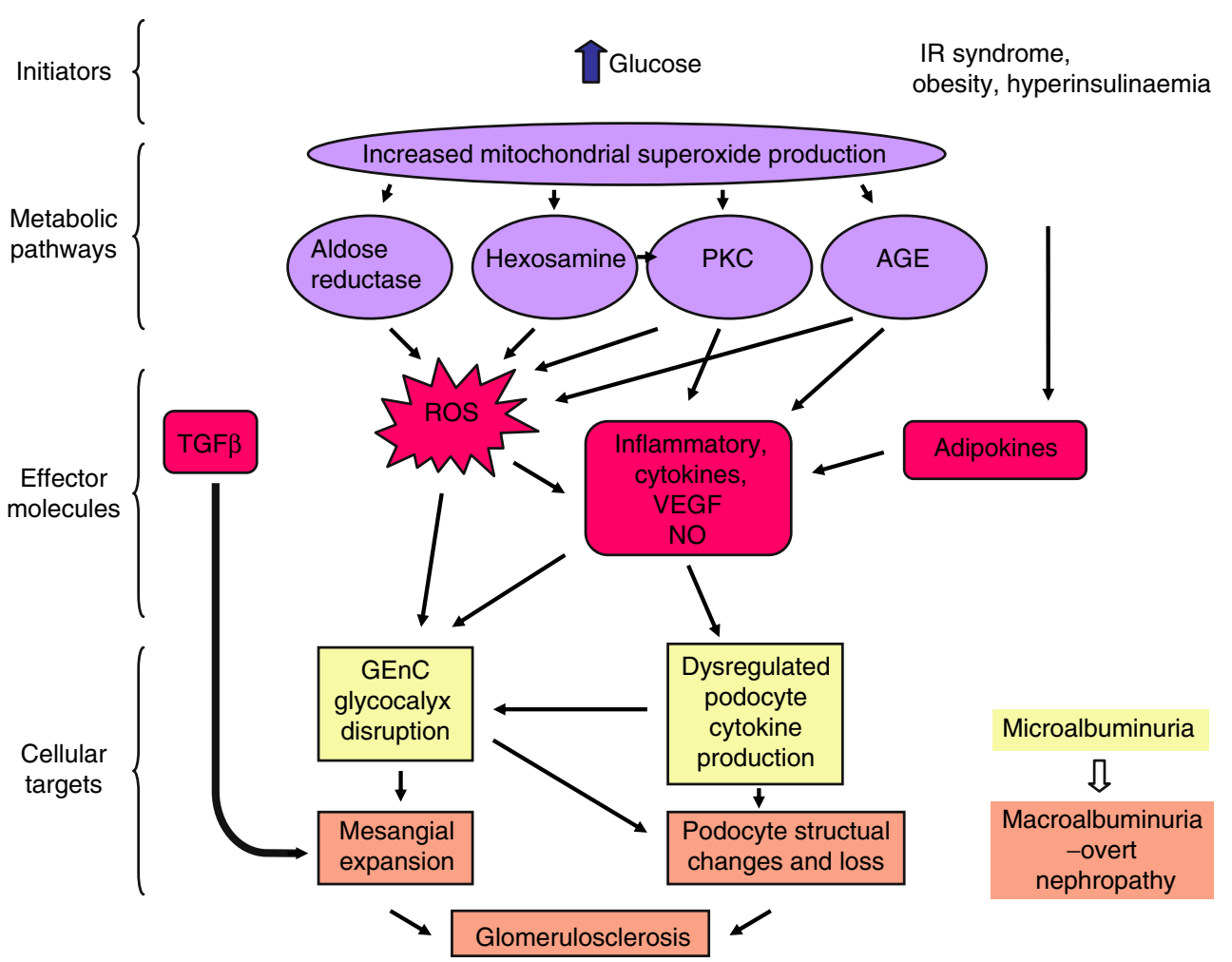

part, dissection of these pathways and elucidation of their role in glomerular disease has necessarily relied on tissue culture or animal models. Such are described where human studies are lacking or where they provide additional significant insights.

ROS Brownlee has proposed oxidative stress as a unifying mechanism whereby the above-mentioned four pathways are inter-linked in the pathogenesis of diabetic complications (Fig. 3) [66]. Hyperglycaemia increases oxidative stress through overproduction of superoxide and other ROS by the mitochondrial electron transport chain. ROS have direct cellular effects (e.g. they increase activity of nuclear factor $\mathrm{kB}[\mathrm{NF} \mathrm{kB}]$, a key inflammatory regulator), increase oxidative stress and interact with the above four pathways. Normalisation of mitochondrial superoxide production blocks all four of these pathways implicated in hyperglycaemic damage and corrects a variety of hyperglycaemia-induced phenotypes in target cells of diabetic complications. Endogenous superoxide dismutase normally neutralises excess superoxide but is overwhelmed in the diabetic state.

As well as forming a nexus of metabolic pathways dysregulated by hyperglycaemia, ROS can also be considered as effectors through direct cellular actions. ROS decrease glomerular HSPG production [67], directly disrupt the endothelial glycocalyx [68], interfere with nitric oxide bioavailability and activate NFKB.
Glomerular ROS production is increased in experimental diabetes [69] and transgenic overexpression of superoxide dismutase attenuates renal injury, including increases in AER [70]. A superoxide dismutase mimetic also ameliorates increases in glomerular permeability both in vivo and ex vivo in non-diabetic models [71]. Little work has yet been done on the use of antioxidants in human diabetic nephropathy, but in vitro work confirms the importance of ROS in cells of the GFB. Podocytes produce ROS in response to high glucose [72], and genetic overexpression of superoxide dismutase prevents inhibition of endothelial nitric oxide synthase (eNOS) activity by hyperglycaemia in endothelial cells [73].

Biochemical disturbances important in mediating adverse effects of hyperglycaemia damage the endothelium and/or the glycocalyx.

Vascular endothelial growth factor Vascular endothelial growth factor (VEGF) is a key regulator of vascular permeability and angiogenesis and is implicated in the pathogenesis of diabetic retinal neovascularisation. In the glomerulus it is produced in large amounts by podocytes and is thought to be important in maintaining glomerular endothelial cell fenestrations [74]. In rat models of type 1 diabetes, VEGF is upregulated in podocytes throughout the course of disease [75]. VEGF inhibition attenuates glomer- 
ular hypertrophy and albumin excretion and prevents upregulation of eNOS production in glomerular endothelial cells [76]. Similar effects are observed in some, but not all, models of type 2 diabetes [77].

In human type 1 diabetes, serum VEGF concentrations vary according to glycaemic control, and higher levels are associated with microvascular complications, including microalbuminuria [78]. In type 2 diabetes, VEGF is upregulated early in the course of disease and urinary VEGF levels are correlated with microalbuminuria [79]. Further studies have confirmed initial upregulation of VEGF signalling in type 2 diabetes followed by a downregulation as podocyte loss and sclerosis develops [80].

VEGF has the potential to induce the new vessel growth seen in early diabetic nephropathy [53] and to alter the permeability characteristics of the endothelium. Indeed, there is increasing evidence of the importance of precise control of glomerular VEGF for normal GFB function: both transgenic podocyte-specific under- or overexpression result in glomerular abnormalities, including glomerular endothelial changes and proteinuria [81]. Retardation of albuminuria in experimental diabetes by angiogenesis inhibitors further implies the importance of angiogenesis and/or endothelial-related processes in the development of diabetic microalbuminuria $[52,82]$.

Experimental interventions that protect the endothelium reduce microalbuminuria.

The growth hormone/IGF system Dysregulation of the growth hormone/IGF system can be detected early in experimental diabetes and is associated with both glomerular hypertrophy and microalbuminuria [83]. All components of the growth hormone/IGF system can be detected at the mRNA level in the normal kidney. However, as detailed histochemical studies have not been performed, the exact location of system components with the glomerulus and the likely role of particular cell types cannot be defined.

The rapid renal growth in experimental diabetes is preceded by a rise in the renal concentration of IGF-1 [84]. Somatostatin analogues ameliorate the increase in IGF-1 and renal hypertrophy and reduce AER [85]. In human type 1 diabetes, serum growth hormone levels and urinary IGF-1 levels are elevated and correlate with microalbuminuria, while an IGF-1 gene polymorphism modifies the risk of development of microalbuminuria [86]. The somatostatin analogue octreotide reduces macroalbuminuria and endothelial dysfunction in type 2 diabetes [87].

Taken together, this evidence points to a role for the growth hormone/IGF system early in human diabetic nephropathy. However, there are few clues to its contribution to the pathogenesis of microalbuminuria at the structural level. IGF-1 activates intracellular intermediates, including NFKB, and an IGF-1 receptor inhibitor suppresses VEGF production, suggesting that VEGF is a downstream mediator of the effects of IGF-1 [88].

Proinflammatory cytokines and adipokines TNF $\alpha$ is a proinflammatory cytokine with diverse actions, including increased production of endothelial cell adhesion molecules and IL-6, which in turn regulates CRP. TNF $\alpha$ also directly increases endothelial permeability and disrupts the glycocalyx [41, 89]. In experimental diabetes, TNF $\alpha$ levels rise in urine and the renal interstitium prior to the onset of albuminuria and correlate with it [90], as they do in human type 2 diabetes [91]. IL-6 levels also correlate with albuminuria in type 1 and 2 diabetes [91, 92]. CRP, often thought of as a downstream marker of inflammation, is elevated in both type 1 and 2 diabetes [27, 93] and correlates strongly with cardiovascular disease.

Adipokines, which include leptin and adiponectin (and, arguably, TNF $\alpha$ and IL-6), also have the potential to contribute to the development of microalbuminuria in both non-diabetic and diabetic populations. Enlargement of fat cells in obesity is associated with a generalised proinflammatory state, including increased levels of TNF $\alpha$ and IL-6, and hypersecretion of adipokines, with the exception of adiponectin, which is downregulated [94]. Leptin induces vascular permeability and synergistically stimulates angiogenesis with VEGF [95]. Its serum levels correlate strongly with nephropathy in type 2 diabetes [96]. Adiponectin, unlike other adipokines, appears to have protective effects in the vasculature in general by reducing endothelial cell activation and inflammation.

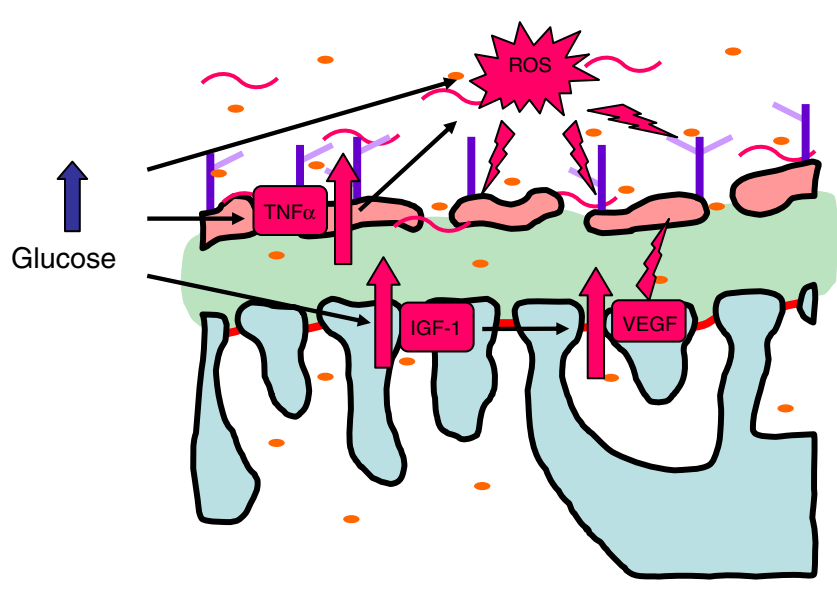

Fig. 4 Proposed mechanism of glomerular filtration barrier damage leading to diabetic microalbuminuria. High glucose causes dysregulation of mediators including TNF $\alpha$ and enhanced production of ROS, which directly damage the glomerular endothelial glycocalyx leading to microalbuminuria. Increased levels of pro-angiogenic molecules, including VEGF and inflammatory mediators, induce an activated and more permeable glomerular endothelial cell phenotype 
What do these changes in regulation and expression of these mediators tell us about the mechanism of microalbuminuria? It is clear that ROS and oxidative stress have a central role, given their importance in various metabolic pathways and direct cellular effects, including in the disruption of endothelial glycocalyx, which may be relevant in pathogenesis of microalbuminuria. There is strong evidence for a role of VEGF early in the course of diabetic nephropathy, probably through upregulation of production by podocytes and actions on glomerular endothelial cells disrupting their contribution to the GFB. The importance of dysregulation of angiogenic factors is emphasised by the protective effects of angiogenesis inhibitors. The growth hormone/IGF system is also dysregulated early in diabetes, and it appears that IGF-1 is an important contributor to microalbuminuria through VEGF. Involvement of inflammatory mediators again points to a role for glomerular endothelial cells through endothelial activation, and hence, an increase in permeability through disruption of the glycocalyx.

\section{Conclusions}

In summary, the various avenues of study of diabetic microalbuminuria reviewed converge on the glomerular endothelium. Our analysis therefore leads to the conclusion that this is the site of the initial damage that leads to the development of microalbuminuria in diabetes. The most important aspect of this damage is disruption of the endothelial glycocalyx through actions of mediators dysregulated by the diabetic milieu. Key players include ROS, VEGF and proinflammatory cytokines (Fig. 4). Disturbance of endothelial cell-podocyte communication contributes to and amplifies the endothelial lesion. Progression of microalbuminuria to overt nephropathy is accompanied by predictable structural changes in the glomerulus, including podocyte damage and loss. This is the result of the ongoing diabetic milieu and disturbed cell-cell communication, but is also secondary to increased penetration of the GFB by serum proteins [97].

Thus recent evidence remains broadly supportive of the Steno hypothesis [4] but it points specifically to disturbance of the endothelial glycocalyx as the common process underlying both microalbuminuria and generalised endothelial dysfunction. While it would be overly simplistic, particularly in type 2 diabetes, to suggest that this is the only factor involved (Fig. 4), it follows nevertheless that microalbuminuria is an indicator of generalised endothelial dysfunction. Most interestingly, these conclusions imply that glycocalyx dysfunction is involved in the pathogenesis of other vascular disease, both microvascular and macrovascular. Therefore, therapies aimed at protecting or repairing endothelial cells and their glycocalyx would be expected to retard these diseases. Reduction or reversal of microalbuminuria implies resolution of generalised endothelial dysfunction and has potential to be a useful indicator of successful reduction of overall cardiovascular risk [98].

Acknowledgements S. C. Satchell is funded by a Wellcome Trust fellowship (075731). We thank F. Holleman for helpful suggestions on preparation of the manuscript.

Duality of interest The authors have no conflicts of interest to declare

Open Access This article is distributed under the terms of the Creative Commons Attribution Noncommercial License which permits any noncommercial use, distribution, and reproduction in any medium, provided the original author(s) and source are credited.

\section{References}

1. Stehouwer CD, Smulders YM (2006) Microalbuminuria and risk for cardiovascular disease: Analysis of potential mechanisms. J Am Soc Nephrol 17:2106-2111

2. Warram JH, Gearin G, Laffel L, Krolewski AS (1996) Effect of duration of type I diabetes on the prevalence of stages of diabetic nephropathy defined by urinary albumin/creatinine ratio. J Am Soc Nephrol 7:930-937

3. Mogensen CE, Poulsen PL (1994) Epidemiology of microalbuminuria in diabetes and in the background population. Curr Opin Nephrol Hypertens 3:248-256

4. Deckert T, Feldt-Rasmussen B, Borch-Johnsen K, Jensen T, Kofoed-Enevoldsen A (1989) Albuminuria reflects widespread vascular damage. The Steno hypothesis. Diabetologia 32:219-226

5. Parving HH (1996) Microalbuminuria in essential hypertension and diabetes mellitus. J Hypertens Suppl 14:S89-S93 discussion S93-S84

6. Bianchi S, Bigazzi R, Campese VM (1999) Microalbuminuria in essential hypertension: significance, pathophysiology, and therapeutic implications. Am J Kidney Dis 34:973-995

7. Jensen JS, Feldt-Rasmussen B, Strandgaard S, Schroll M, BorchJohnsen K (2000) Arterial hypertension, microalbuminuria, and risk of ischemic heart disease. Hypertension 35:898-903

8. Schalkwijk CG, Stehouwer CD (2005) Vascular complications in diabetes mellitus: the role of endothelial dysfunction. Clin Sci (Lond) 109:143-159

9. De Gaudio AR, Adembri C, Grechi S, Novelli GP (2000) Microalbuminuria as an early index of impairment of glomerular permeability in postoperative septic patients. Intensive Care Med 26:1364-1368

10. Mahmud N, O'Connell MA, Stinson J, Goggins MG, Weir DG, Kelleher D (1995) Tumour necrosis factor-alpha and microalbuminuria in patients with inflammatory bowel disease. Eur $\mathrm{J}$ Gastroenterol Hepatol 7:215-219

11. Hillege HL, Janssen WM, Bak AA et al (2001) Microalbuminuria is common, also in a nondiabetic, nonhypertensive population, and an independent indicator of cardiovascular risk factors and cardiovascular morbidity. J Intern Med 249:519-526

12. Monster TB, Janssen WM, de Jong PE, de Jong-van den Berg LT (2001) Oral contraceptive use and hormone replacement therapy are associated with microalbuminuria. Arch Intern Med 161:2000-2005

13. Sowers JR, Epstein M, Frohlich ED (2001) Diabetes, hypertension, and cardiovascular disease: an update. Hypertension 37:1053-1059 
14. Hovind P, Rossing P, Tarnow L, Smidt UM, Parving HH (2001) Remission and regression in the nephropathy of type 1 diabetes when blood pressure is controlled aggressively. Kidney Int $60: 277-283$

15. Chaturvedi N, Bandinelli S, Mangili R, Penno G, Rottiers RE, Fuller JH (2001) Microalbuminuria in type 1 diabetes: rates, risk factors and glycemic threshold. Kidney Int 60:219-227

16. Quinn M, Angelico MC, Warram JH, Krolewski AS (1996) Familial factors determine the development of diabetic nephropathy in patients with IDDM. Diabetologia 39:940-945

17. Campos-Pastor MM, Escobar-Jimenez F, Mezquita P et al (2000) Factors associated with microalbuminuria in type 1 diabetes mellitus: a cross-sectional study. Diabetes Res Clin Pract 48: 43-49

18. Yip J, Mattock MB, Morocutti A, Sethi M, Trevisan R, Viberti G (1993) Insulin resistance in insulin-dependent diabetic patients with microalbuminuria. Lancet 342:883-887

19. Rossing P, Hougaard P, Borch-Johnsen K, Parving HH (1996) Predictors of mortality in insulin dependent diabetes: 10 year observational follow up study. BMJ 313:779-784

20. Dinneen SF, Gerstein HC (1997) The association of microalbuminuria and mortality in non-insulin- dependent diabetes mellitus. A systematic overview of the literature. Arch Intern Med 157:1413-1418

21. Borch-Johnsen K, Feldt-Rasmussen B, Strandgaard S, Schroll M, Jensen JS (1999) Urinary albumin excretion. An independent predictor of ischemic heart disease. Arterioscler Thromb Vasc Biol 19:1992-1997

22. Fioretto P, Stehouwer CD, Mauer M et al (1998) Heterogeneous nature of microalbuminuria in NIDDM: studies of endothelial function and renal structure. Diabetologia 41:233-236

23. Adler AI, Stevens RJ, Manley SE, Bilous RW, Cull CA, Holman RR (2003) Development and progression of nephropathy in type 2 diabetes: the United Kingdom Prospective Diabetes Study (UKPDS 64). Kidney Int 63:225-232

24. Parving HH, Chaturvedi N, Viberti G, Mogensen CE (2002) Does microalbuminuria predict diabetic nephropathy. Diabetes Care 25: 406-407

25. Gerstein HC, Mann JF, Yi Q et al (2001) Albuminuria and risk of cardiovascular events, death, and heart failure in diabetic and nondiabetic individuals. JAMA 286:421-426

26. Stehouwer CD, Henry RM, Dekker JM, Nijpels G, Heine RJ, Bouter LM (2004) Microalbuminuria is associated with impaired brachial artery, flow-mediated vasodilation in elderly individuals without and with diabetes: further evidence for a link between microalbuminuria and endothelial dysfunction - the Hoorn Study. Kidney Int Suppl:S42-S44

27. Schalkwijk CG, Poland DC, van Dijk W et al (1999) Plasma concentration of C-reactive protein is increased in type I diabetic patients without clinical macroangiopathy and correlates with markers of endothelial dysfunction: evidence for chronic inflammation. Diabetologia 42:351-357

28. Willerson JT, Ridker PM (2004) Inflammation as a cardiovascular risk factor. Circulation 109:II2-II10

29. Stehouwer CD, Fischer HR, van Kuijk AW, Polak BC, Donker AJ (1995) Endothelial dysfunction precedes development of microalbuminuria in IDDM. Diabetes 44:561-564

30. Stehouwer CD, Gall MA, Twisk JW, Knudsen E, Emeis JJ, Parving HH (2002) Increased urinary albumin excretion, endothelial dysfunction, and chronic low-grade inflammation in type 2 diabetes: progressive, interrelated, and independently associated with risk of death. Diabetes 51:1157-1165

31. Deen WM, Lazzara MJ, Myers BD (2001) Structural determinants of glomerular permeability. Am J Physiol Renal Physiol 281:F579-F596

32. Ballermann BJ (2007) Contribution of the endothelium to the glomerular permselectivity barrier in health and disease. Nephron Physiol 106:p19-p25
33. Rostgaard J, Qvortrup K (1997) Electron microscopic demonstrations of filamentous molecular sieve plugs in capillary fenestrae. Microvasc Res 53:1-13

34. Hjalmarsson C, Johansson BR, Haraldsson B (2004) Electron microscopic evaluation of the endothelial surface layer of glomerular capillaries. Microvasc Res 67:9-17

35. Huxley VH, Williams DA (2000) Role of a glycocalyx on coronary arteriole permeability to proteins: evidence from enzyme treatments. Am J Physiol Heart Circ Physiol 278:H1177-H1185

36. Rehm M, Zahler S, Lotsch M et al (2004) Endothelial glycocalyx as an additional barrier determining extravasation of $6 \%$ hydroxyethyl starch or $5 \%$ albumin solutions in the coronary vascular bed. Anesthesiology 100:1211-1223

37. Levick JR, Smaje LH (1987) An analysis of the permeability of a fenestra. Microvasc Res 33:233-256

38. Ohlson M, Sorensson J, Haraldsson B (2001) A gel-membrane model of glomerular charge and size selectivity in series. Am J Physiol Renal Physiol 280:F396-H405

39. Jeansson M, Haraldsson B (2006) Morphological and functional evidence for an important role of the endothelial cell glycocalyx in the glomerular barrier. Am J Physiol Renal Physiol 290:F111-H116

40. Ryan GB, Karnovsky MJ (1976) Distribution of endogenous albumin in the rat glomerulus: role of hemodynamic factors in glomerular barrier function. Kidney Int 9:36-45

41. Henry CB, Duling BR (2000) TNF-alpha increases entry of macromolecules into luminal endothelial cell glycocalyx. Am J Physiol Heart Circ Physiol 279:H2815-H2823

42. Yoshioka T, Ichikawa I, Fogo A (1991) Reactive oxygen metabolites cause massive, reversible proteinuria and glomerular sieving defect without apparent ultrastructural abnormality. J Am Soc Nephrol 2:902-912

43. Singh A, Satchell SC, Neal CR, McKenzie EA, Tooke JE, Mathieson PW (2007) Glomerular endothelial glycocalyx constitutes a barrier to protein permeability. J Am Soc Nephrol 18:2885-2893

44. Pavenstadt H, Kriz W, Kretzler M (2003) Cell biology of the glomerular podocyte. Physiol Rev 83:253-307

45. Haraldsson B, Sorensson J (2004) Why do we not all have proteinuria? An update of our current understanding of the glomerular barrier. News Physiol Sci 19:7-10

46. Drumond MC, Deen WM (1994) Structural determinants of glomerular hydraulic permeability. Am J Physiol 266:F1-F12

47. Lazzara MJ, Deen WM (2007) Model of albumin reabsorption in the proximal tubule. Am J Physiol Renal Physiol 292:F430-F439

48. Ohlson M, Sorensson J, Lindstrom K, Blom AM, Fries E, Haraldsson B (2001) Effects of filtration rate on the glomerular barrier and clearance of four differently shaped molecules. Am J Physiol Renal Physiol 281:F103-F113

49. Dalla Vestra M, Saller A, Bortoloso E, Mauer M, Fioretto P (2000) Structural involvement in type 1 and type 2 diabetic nephropathy. Diabetes Metab 26(Suppl 4):8-14

50. Dalla Vestra M, Masiero A, Roiter AM, Saller A, Crepaldi G, Fioretto P (2003) Is podocyte injury relevant in diabetic nephropathy? Studies in patients with type 2 diabetes. Diabetes 52:1031-1035

51. Pagtalunan ME, Miller PL, Jumping-Eagle S et al (1997) Podocyte loss and progressive glomerular injury in type II diabetes. J Clin Invest 99:342-348

52. Osterby R, Asplund J, Bangstad HJ et al (1999) Neovascularization at the vascular pole region in diabetic glomerulopathy. Nephrol Dial Transplant 14:348-352

53. Kanesaki Y, Suzuki D, Uehara G et al (2005) Vascular endothelial growth factor gene expression is correlated with glomerular neovascularization in human diabetic nephropathy. Am J Kidney Dis 45:288-294

54. Nieuwdorp M, van Haeften TW, Gouverneur MC et al (2006) Loss of endothelial glycocalyx during acute hyperglycemia 
coincides with endothelial dysfunction and coagulation activation in vivo. Diabetes 55:480-486

55. Nieuwdorp M, Mooij HL, Kroon J et al (2006) Endothelial glycocalyx damage coincides with microalbuminuria in type 1 diabetes. Diabetes 55:1127-1132

56. van den Berg JG, van den Bergh Weerman MA, Assmann KJ, Weening JJ, Florquin S (2004) Podocyte foot process effacement is not correlated with the level of proteinuria in human glomerulopathies. Kidney Int 66:1901-1906

57. Karumanchi SA, Epstein FH, Stillman IE (2005) Is loss of podocyte foot processes necessary for the induction of proteinuria. Am J Kidney Dis 45:436

58. Lemley KV, Blouch K, Abdullah I et al (2000) Glomerular permselectivity at the onset of nephropathy in type 2 diabetes mellitus. J Am Soc Nephrol 11:2095-2105

59. White KE, Bilous RW, Marshall SM et al (2002) Podocyte number in normotensive type 1 diabetic patients with albuminuria. Diabetes 51:3083-3089

60. Carraro M, Mancini W, Artero M et al (2000) Albumin permeability in isolated glomeruli in incipient experimental diabetes mellitus. Diabetologia 43:235-241

61. Jeansson M, Granqvist AB, Nystrom JS, Haraldsson B (2006) Functional and molecular alterations of the glomerular barrier in long-term diabetes in mice. Diabetologia 49:2200-2209

62. Jensen JS, Borch-Johnsen K, Deckert T, Deckert M, Jensen G, Feldt-Rasmussen B (1995) Reduced glomerular size- and chargeselectivity in clinically healthy individuals with microalbuminuria. Eur J Clin Invest 25:608-614

63. Deckert T, Kofoed-Enevoldsen A, Vidal P, Norgaard K, Andreasen HB, Feldt-Rasmussen B (1993) Size- and charge selectivity of glomerular filtration in type 1 (insulin-dependent) diabetic patients with and without albuminuria. Diabetologia 36:244-251

64. Ziyadeh FN, Hoffman BB, Han DC et al (2000) Long-term prevention of renal insufficiency, excess matrix gene expression, and glomerular mesangial matrix expansion by treatment with monoclonal antitransforming growth factor-beta antibody in $\mathrm{db} / \mathrm{db}$ diabetic mice. Proc Natl Acad Sci USA 97:8015-8020

65. Wang A, Ziyadeh FN, Lee EY et al (2007) Interference with TGFbeta signaling by Smad3-knockout in mice limits diabetic glomerulosclerosis without affecting albuminuria. Am J Physiol Renal Physiol 293:F1657-F1665

66. Brownlee M (2001) Biochemistry and molecular cell biology of diabetic complications. Nature 414:813-820

67. Kashihara N, Watanabe Y, Makino H, Wallner EI, Kanwar YS (1992) Selective decreased de novo synthesis of glomerular proteoglycans under the influence of reactive oxygen species. Proc Natl Acad Sci USA 89:6309-6313

68. Vink H, Duling BR (1996) Identification of distinct luminal domains for macromolecules, erythrocytes, and leukocytes within mammalian capillaries. Circ Res 79:581-589

69. Satoh M, Fujimoto S, Haruna Y et al (2005) NAD(P)H oxidase and uncoupled nitric oxide synthase are major sources of glomerular superoxide in rats with experimental diabetic nephropathy. Am J Physiol Renal Physiol 288:F1144-F1152

70. DeRubertis FR, Craven PA, Melhem MF, Salah EM (2004) Attenuation of renal injury in $\mathrm{db} / \mathrm{db}$ mice overexpressing superoxide dismutase: evidence for reduced superoxide-nitric oxide interaction. Diabetes 53:762-768

71. Duann P, Datta PK, Pan C, Blumberg JB, Sharma M, Lianos EA (2006) Superoxide dismutase mimetic preserves the glomerular capillary permeability barrier to protein. J Pharmacol Exp Ther 316:1249-1254

72. Susztak K, Raff AC, Schiffer M, Bottinger EP (2006) Glucoseinduced reactive oxygen species cause apoptosis of podocytes and podocyte depletion at the onset of diabetic nephropathy. Diabetes $55: 225-233$
73. Du XL, Edelstein D, Dimmeler S, Ju Q, Sui C, Brownlee M (2001) Hyperglycemia inhibits endothelial nitric oxide synthase activity by posttranslational modification at the Akt site. J Clin Invest 108:1341-1348

74. Satchell SC, Tasman CH, Singh A et al (2006) Conditionally immortalized human glomerular endothelial cells expressing fenestrations in response to VEGF. Kidney Int 69:1633-1640

75. Cooper ME, Vranes D, Youssef S et al (1999) Increased renal expression of vascular endothelial growth factor (VEGF) and its receptor VEGFR-2 in experimental diabetes. Diabetes 48:2229-2239

76. de Vriese AS, Tilton RG, Elger M, Stephan CC, Kriz W, Lameire NH (2001) Antibodies against vascular endothelial growth factor improve early renal dysfunction in experimental diabetes. J Am Soc Nephrol 12:993-1000

77. Flyvbjerg A, Dagnaes-Hansen F, De Vriese AS, Schrijvers BF, Tilton RG, Rasch R (2002) Amelioration of long-term renal changes in obese type 2 diabetic mice by a neutralizing vascular endothelial growth factor antibody. Diabetes 51:3090-3094

78. Chiarelli F, Spagnoli A, Basciani F et al (2000) Vascular endothelial growth factor (VEGF) in children, adolescents and young adults with type 1 diabetes mellitus: relation to glycaemic control and microvascular complications. Diabet Med 17:650-656

79. Kim NH, Oh JH, Seo JA et al (2005) Vascular endothelial growth factor (VEGF) and soluble VEGF receptor FLT-1 in diabetic nephropathy. Kidney Int 67:167-177

80. Hohenstein B, Hausknecht B, Boehmer K, Riess R, Brekken RA, Hugo CP (2006) Local VEGF activity but not VEGF expression is tightly regulated during diabetic nephropathy in man. Kidney Int 69:1654-1661

81. Eremina V, Sood M, Haigh J et al (2003) Glomerular-specific alterations of VEGF-A expression lead to distinct congenital and acquired renal diseases. J Clin Invest 111:707-716

82. Zhang SX, Wang JJ, Lu K, Mott R, Longeras R, Ma JX (2006) Therapeutic potential of angiostatin in diabetic nephropathy. J Am Soc Nephrol 17:475-486

83. Schrijvers BF, De Vriese AS, Flyvbjerg A (2004) From hyperglycemia to diabetic kidney disease: the role of metabolic, hemodynamic, intracellular factors and growth factors/cytokines. Endocr Rev 25:9711010

84. Flyvbjerg A, Bornfeldt KE, Marshall SM, Arnqvist HJ, Orskov H (1990) Kidney IGF-I mRNA in initial renal hypertrophy in experimental diabetes in rats. Diabetologia 33:334-338

85. Gronbaek H, Vogel I, Osterby R, Lancranjan I, Flyvbjerg A, Orskov H (1998) Effect of octreotide, captopril or insulin on renal changes and UAE in long-term experimental diabetes. Kidney Int 53:173-180

86. Hovind P, Lamberts S, Hop W et al (2007) An IGF-I gene polymorphism modifies the risk of developing persistent microalbuminuria in type 1 diabetes. Eur J Endocrinol 156:83-90

87. Clemens A, Klevesath MS, Hofmann M et al (1999) Octreotide (somatostatin analog) treatment reduces endothelial cell dysfunction in patients with diabetes mellitus. Metabolism 48:1236-1240

88. Smith LE, Shen W, Perruzzi C et al (1999) Regulation of vascular endothelial growth factor-dependent retinal neovascularization by insulin-like growth factor-1 receptor. Nat Med 5:1390-1395 (java/ Propub/medicine/nm1299 1390.fulltext java/Propub/medicine/ nm1299 1390.abstract)

89. Friedl J, Puhlmann M, Bartlett DL et al (2002) Induction of permeability across endothelial cell monolayers by tumor necrosis factor (TNF) occurs via a tissue factor-dependent mechanism: relationship between the procoagulant and permeability effects of TNF. Blood 100:1334-1339

90. Navarro JF, Milena FJ, Mora C et al (2005) Tumor necrosis factor-alpha gene expression in diabetic nephropathy: relationship with urinary albumin excretion and effect of angiotensin-converting enzyme inhibition. Kidney Int 68:S98-S102 
91. Moriwaki Y, Yamamoto T, Shibutani Y et al (2003) Elevated levels of interleukin-18 and tumor necrosis factor-alpha in serum of patients with type 2 diabetes mellitus: relationship with diabetic nephropathy. Metabolism 52:605-608

92. Saraheimo M, Teppo AM, Forsblom C, Fagerudd J, Groop PH (2003) Diabetic nephropathy is associated with low-grade inflammation in type 1 diabetic patients. Diabetologia 46:1402-1407

93. McMillan DE (1989) Increased levels of acute-phase serum proteins in diabetes. Metabolism 38:1042-1046

94. Yang X, Smith U (2007) Adipose tissue distribution and risk of metabolic disease: does thiazolidinedione-induced adipose tissue redistribution provide a clue to the answer? Diabetologia 50:1127-1139
95. Cao R, Brakenhielm E, Wahlestedt C, Thyberg J, Cao Y (2001) Leptin induces vascular permeability and synergistically stimulates angiogenesis with FGF-2 and VEGF. Proc Natl Acad Sci U S A 98:6390-6395

96. Matsuda M, Kawasaki F, Yamada K et al (2004) Impact of adiposity and plasma adipocytokines on diabetic angiopathies in Japanese Type 2 diabetic subjects. Diabet Med 21:881-888

97. Williams ME (2005) Diabetic nephropathy: the proteinuria hypothesis. Am J Nephrol 25:77-94

98. Jensen JS, Borch-Johnsen K, Jensen G, Feldt-Rasmussen B (1995) Microalbuminuria reflects a generalized transvascular albumin leakiness in clinically healthy subjects. Clin Sci (Colch) 88:629-633 